\title{
Cerebroventricular Microinjections of MPTP on Adult Zebrafish Induces Dopaminergic Neuronal Death, Mitochondrial Fragmentation, and Sensorimotor Impairments
}

\author{
Michael Kalyn and Marc Ekker* \\ Department of Biology, Faculty of Science, University of Ottawa, Ottawa, ON, Canada
}

OPEN ACCESS

Edited by:

Maria Xilouri,

Biomedical Research Foundation

of the Academy of Athens, Greece

Reviewed by:

Oeystein Roed Brekk,

Harvard Medical School,

United States

Natalie Landeck,

National Institute on Aging, National

Institutes of Health (NIH),

United States

*Correspondence:

Marc Ekker

mekker@uottawa.ca

Specialty section:

This article was submitted to

Neurodegeneration,

a section of the journal

Frontiers in Neuroscience

Received: 31 May 2021

Accepted: 26 July 2021

Published: 27 August 2021

Citation:

Kalyn M and Ekker M (2021) Cerebroventricular Microinjections of MPTP on Adult Zebrafish Induces

Dopaminergic Neuronal Death,

Mitochondrial Fragmentation,

and Sensorimotor Impairments.

Front. Neurosci. 15:718244.

doi: 10.3389/fnins.2021.718244
Mitochondria are dynamic organelles that mediate the energetic supply to cells and mitigate oxidative stress through the intricate balance of fission and fusion. Mitochondrial dysfunction is a prominent feature within Parkinson disease (PD) etiologies. To date, there have been conflicting studies of neurotoxin impact on dopaminergic cell death, mitochondrial function and behavioral impairment using adult zebrafish. Here, we performed cerebroventricular microinjections (CVMIs) of 1-methyl-4-phenyl-1,2,3,6tetrahydropyridine (MPTP) on adult transgenic zebrafish that resulted in significant reductions in dopaminergic neurons within the telencephalon and olfactory bulbs (OB) of Tg(dat:eGFP) fish. Visualization of mCherry and mitochondrial gene expression analysis in Tg(dat:tom20 MLS:mCherry) fish reveal that MPTP induces mitochondrial fragmentation in dopaminergic neurons and the activation of the pink1/parkin pathway involved mitophagy. Moreover, the loss of dopaminergic neurons translated into a transient locomotor and olfactory phenotype. Taken together, these data can contribute to a better understanding of the mitochondrial impact on dopaminergic survivability.

Keywords: Parkinson's disease, zebrafish, regeneration, dopamine neuron, neurotoxin, drug administration

\section{INTRODUCTION}

Idiopathic in nature, Parkinson disease (PD) is the second most prevalent neurodegenerative disease in the world where symptoms comprise of motor, sensory and cognitive impairments (de Lau and Breteler, 2006; Muangpaisan et al., 2011). The pathology of PD has been characterized as a progressive loss of dopaminergic neurons within the substantia nigra of the human midbrain (Dauer and Przedborski, 2003; de Lau and Breteler, 2006; Muangpaisan et al., 2011). Motor and sensory symptoms develop following 40-60\% loss of these neurons (Cheng et al., 2010).

Parkinson disease is believed to be a cumulative result of genetic and environmental insult. Researchers have elucidated mutations in various genes [PINK1 (Hatano et al., 2004), Parkin (Kitada et al., 1998; Dawson and Dawson, 2010), LRRK2 (Funayama et al., 2002; Zimprich et al., 2004), and DJ-1(Bonifati et al., 2003)] that increase an individual's susceptibility to developing PD. However, this can only explain $5-10 \%$ of cases, while the majority are sporadically induced through external factors such as: age, gender, neurotoxic exposure, and mitochondrial dysfunction (Dexter and Jenner, 2013).

Chemo-ablative models for PD commonly consist of the administration or exposure to 1methyl-4-phenyl-1,2,3,6-tetrahydropyridine (MPTP; Meredith and Rademacher, 2011; Tieu, 2011; 
Hare et al., 2013). Being lipophilic, MPTP readily permeates the blood-brain-barrier and is metabolized into its active toxic form $\mathrm{MPP}+$ through monoamine oxidase-B (MAO-B) in proximal astrocytes (Przedborski et al., 2004; Hare et al., 2013). $\mathrm{MPP}+$ enters dopaminergic neurons through the dopamine transporter (Dat) protein to exert its cytotoxicity through the inhibition of complex I activity in the electron transport chain (ETC) of mitochondria (Przedborski et al., 2004). MPTP has been extensively used in PD studies in a variety of models ranging from mice, rats, primates, and zebrafish (Van Kampen et al., 2000; Meredith and Rademacher, 2011; Bové and Perier, 2012). Previous studies in zebrafish have been carried out using embryonic or larval stages in development. These studies have demonstrated the effect of MPTP on locomotion, morphology, dopaminergic survivability and have identified various neuroprotective compounds (Lam et al., 2005; Sallinen et al., 2009; Díaz-Casado et al., 2016; Kalyn et al., 2019; Zhao et al., 2020). However, studies pertaining to MPTP exposure in adult zebrafish address the behavioral phenotype but commonly disregard or report inconsistencies surrounding the impact on sensory perception, dopaminergic neurons and their mitochondria (Anichtchik et al., 2004; Bretaud et al., 2004).

Many studies have adopted the use of mammalian models to study $\mathrm{PD}$, however, the use of zebrafish has rapidly emerged and proven to be considerably beneficial. Zebrafish possess transparent ex utero embryogenesis allowing for indepth developmental imaging, as well as, large clutch sizes following breeding to facilitate high-throughput drug screening (Stewart et al., 2014). This model also displays many conserved pathologies and molecular pathways due to their genome being $\sim 87 \%$ homologous to humans (Du et al., 2016). Pertinent to $\mathrm{PD}$, zebrafish possess a functional analogue to the dopaminergic nigrostriatal system described in humans (Holzschuh et al., 2001; Sallinen et al., 2009).

Given the accessibility of zebrafish embryos into adulthood, this model is widely used for pharmacological studies. Conventional drug delivery methods comprise of direct immersion, intraperitoneal (IP) injections and intramuscular (IM) injections, however, there are significant pitfalls to each. In this study, we optimized a drug delivery method through cerebroventricular microinjections (CVMIs) to administer MPTP to adult zebrafish. MPTP is shown to selectively ablate dopaminergic populations through mitophagy mediated cell death demonstrated through increased mitochondrial fragmentation, as observed in adult $\mathrm{Tg}$ (dat:tom 20 MLS:mCherry) zebrafish. This effect was complimented with gene expression analyses of mitochondrial regulatory genes. Moreover, this decrease in dopaminergic neurons translates into transient sensorimotor perturbing phenotypes.

\section{MATERIALS AND METHODS}

\section{Zebrafish Care and Husbandry}

The transgenic zebrafish lines used for this study are $\operatorname{Tg}($ dat:eGFP) and $\operatorname{Tg}($ dat:tom 20 MLS:mCherry) (Xi et al., 2011; Noble et al., 2015). Embryos were collected by natural spawning to be raised in E3 embryo media $(13 \mathrm{mM} \mathrm{NaCl}$, $0.5 \mathrm{mM} \mathrm{KCL}, 0.02 \mathrm{mM} \mathrm{Na}_{2} \mathrm{HPO}_{4}, 0.04 \mathrm{mM} \mathrm{KH} \mathrm{PO}_{4}, 1.3 \mathrm{mM}$ $\mathrm{CaCl} 2,1 \mathrm{mM} \mathrm{MgSO}_{4}$, and $4.2 \mathrm{mM} \mathrm{NaHCO}_{3}$ ). Zebrafish were sorted by fluorescent intensity detected at $4 \mathrm{dpf}$ and raised into adulthood for experimentation. All procedures were approved by the University of Ottawa Animal care committee and conducted under the Animal Care and Veterinary Service guidance in accordance with the Canadian council for Animal care following protocol number BL-2081.

\section{1-Methyl-4-Phenyl-1,2,3,6- Tetrahydropyridine Preparation and CVMIs in Adult Zebrafish}

1-Methyl-4-phenyl-1,2,3,6-tetrahydropyridine $\quad\left(\mathrm{C}_{12} \mathrm{H}_{15} \mathrm{~N} \cdot \mathrm{HCl}\right.$, Product: M0896, CAS: 23007-85-4, Sigma, Oakville, ON, Canada) was reconstituted in distilled water to a stock concentration of $500 \mathrm{mM}$ and diluted to the working concentrations of 10 , 25,35 , and $100 \mathrm{mM}$.

Adult $\operatorname{Tg}($ dat:eGFP) and $\operatorname{Tg}$ (dat:tom20 MLS:mCherry) zebrafish ( $\sim 10$-month-old) were anesthetized with Tricaine. In accordance with the procedure outlined in Kizil et al., a small incision of $200 \mathrm{um}$ was then generated with a 30-gauge syringe (BD Ultra-fine II, BD Biosciences) in the cranium above the anterior portion of the optic tectum without damaging the brain. The prepared MPTP was loaded into a thin glass capillary microinjecting needle (Sutter Instrument) to inject the contents through the incision into the cerebroventricular fluid surrounding the brain. Microinjections were performed using the IM-300 vacuum pump microinjector station (Narishige) at a pressure range of 55-65 psi. Phenol Red (Sigma) was co-administered to ensure proper distribution throughout the cerebroventricular fluid (CVF).

\section{Immunohistochemistry on Zebrafish Cryosections}

Zebrafish heads were collected and fixed in a $4 \%$ paraformaldehyde (PFA) solution dissolved in $1 \times$ phosphate buffered saline (PBS) overnight at $4^{\circ} \mathrm{C}$. Brain-dissections were conducted in $1 \times$ PBS and washed thrice in PBS-T followed by equilibration in $30 \%$ sucrose/PBS overnight at $4^{\circ} \mathrm{C}$. The brains were flash frozen in Tissue-tek OCT (VWR) for coronal cryosectioning. Section thickness performed range from 16 to $20 \mu \mathrm{m}$ and were stored at $-20^{\circ} \mathrm{C}$.

Sections were thawed for $30 \mathrm{~min}$ prior to respective antigen retrieval. Sections were placed in $0.05 \%$ sodium citrate in $1 \times$ PBS-T for $15 \mathrm{~min}$ at $85^{\circ} \mathrm{C}$ then cooled at RT for $15 \mathrm{~min}$. Slides were then washed with $1 \times$ PBS twice prior to rehydration in $1 \times$ PBS-T for $10 \mathrm{~min}$. Blocking was then performed, and the slides were incubated with primary antibodies diluted in $1 \%$ fetal bovine serum (FBS) in PBS-T overnight at $4^{\circ} \mathrm{C}$. Primary antibodies used were; anti-GFP (polyclonal rabbit IgG, A11122, Invitrogen) and anti-DsRed (polyclonal rabbit IgG, \#632496, Clontech). Slides were washed and incubated with the secondary antibodies for $2 \mathrm{~h}$ at RT in a dark chamber. Secondary antibodies used were; goat anti-rabbit Alexa 488 conjugate (A11008, Invitrogen) and goat anti-rabbit Alexa 594 conjugate 
(A11012, Invitrogen). Slides were then washed in $1 \times$ PBS-T prior to mounting with DAPI-infused Vectashield mounting media (Lynx Biosciences). Images for eGFP + quantification were captured with the Olympus FV1000 Confocal microscope using a $20 \times$ air immersion objective and images for mCherry+ quantification were captured using a Zeiss LSM 880 Confocal microscope using a $63 \times$ oil immersion objective. Mitochondria were ranked according to their morphology. Fused refers to uniformly linked mitochondria surrounding the nucleus. Partial refers to mitochondrial fragmentations observed in less than 50\% of the mitochondria within the field of view. Fragmented refers mitochondria that appear scattered $(>50 \%)$, truncated and less dense surrounding the nucleus. Counts were performed using Image J software and were performed by two to three independent researchers in a blinded approach to remove personal bias.

\section{Whole Brain Real-Time Quantitative Reverse Transcription PCR (qRT-PCR)}

RNA was extracted from homogenized adult whole zebrafish brains using TRIzol in accordance to the manufacturer's protocol (Invitrogen, Thermo Fisher Scientific, Waltham, MA, United States). The purity and integrity of RNA was determined using the NanoDrop 1000 spectrophotometer (Thermo Fisher Scientific, Waltham, MA, United States) and through gel electrophoresis. Samples only with absorbance of 1.8-2.1 and clear $18 \mathrm{~S}$ and $28 \mathrm{~S}$ bands were used for cDNA synthesis. The reverse-transcription was performed using the iScript cDNA Synthesis Kit following the supplier's protocol. qRTPCR reactions were done in triplicates composed of $5 \mu \mathrm{L}$ SsoFast ${ }^{\mathrm{TM}}$ EvaGreen $^{\circledR}$ Supermix (Bio-Rad), $0.4 \mu \mathrm{L}$ forward primer, $0.4 \mu \mathrm{L}$ reverse primer, $0.2 \mu \mathrm{L}$ nuclease-free water, and $4 \mu \mathrm{L}$ cDNA. All readings were analyzed using the BioRad CFX96 instrument. Relative quantification for th1, dat, p53, pink1, parkin, mao-b, fis1, mfn1, opa1, parla, sox2, and nestin transcripts was obtained through the comparative $\mathrm{Cq}$ method using the following reference genes; ribosomal protein l13a (rpl13a), elongation factor 1 alpha (ef1a), and tyrosine 3monooxygenase/tryptophan 5-monooxygenase activation protein, zeta polypeptide (ywhaz). Oligonucleotide primer sequences are listed in Table 1.

\section{Locomotion Analysis}

Following treatment and recovery, adult zebrafish were monitored for swimming activity. Fish were placed individually in static tanks and allowed to acclimate in the ambient light for 15 min prior to the trials. Recordings were taken using the ZebraCube tracking system (ViewPoint Life Sciences, Lyon, France) and analyzed with Zebralab software. The swimming parameters examined in this study consist of total distance traveled, average swimming velocity and freezing bout duration over the course of 7 min trials. Sample sizes of 10 were selected to delineate the effects of individual swimming variability. Additionally, the same fish were analyzed from the last injection and the recovery period.

\section{Olfactory Analysis}

Following treatment and recovery, adult zebrafish were placed in a customized static tank that is comprised of a midline division to result in a neutral zone, left and right arm (Hussain et al., 2013). Fish were allowed 15 min for tank acclimation in the ambient ZebraCube lighting. Following acclimation, cadaverine (Sigma Aldrich) was added to the arm the fish was in at that time-point referred to as the stimulus arm. Trials of 3 min were conducted to limit cadaverine diffusion into the non-stimulus arm or neutral zone. Data was captured using the ZebraCube tracking system and interpreted with the Zebralab software.

\section{Statistical Analysis}

All statistical analysis was performed using the software GraphPad Prism v7.0 (San Diego, CA, United States). Swimming parameters were examined for $n=10$ adult zebrafish from each treatment group, while the olfactory evasion assay evaluated $n=5$. Both data sets were analyzed using multiple $t$-test comparison with significance determined using the Holm-Sidak method. Gene expression analysis was collected from whole brains from $n=3$ of each treatment group. Similarly, significance was quantified using multiple $t$-test and Holm-Sidak analysis.

TABLE 1 | List of primers designed for qRT-PCR.

\begin{tabular}{|c|c|c|c|}
\hline Primer & Forward Sequence $\left(5^{\prime}-3^{\prime}\right)$ & Reverse Sequence $\left(5^{\prime}-3^{\prime}\right)$ & References \\
\hline rp/13a & TCTGGAGGACTGTAAGAGGTATGC & AGACGCACAATCTTGAGAGCAG & \\
\hline Dat & AGACATCTGGGAAGGTGGTG & ACCTGAGCATCATACAGGCG & Barreto-Valer et al., 2012 \\
\hline th1 & GACGGAAGATGATCGGAGACA & CCGCCATGTTCCGATTTCT & Chen et al., 2016 \\
\hline p53 & ATATCCTGGCGAACATTTGG & ACGTCCACCACCACCATTTGAAC & \\
\hline fis 1 & CCCTGAACCTTCCAGTGTT & GTCTCTGGAAACGGGTCCTT & \\
\hline opa1 & GCTTGAGCGCTTGGAAAAGGAA & TGGCAGGTGATCTTGAGTGTTGT & \\
\hline$m f n 1$ & CTGGGTCCCGTCAACGCCAA & ACTGAACCACCGCTGGGGCT & \\
\hline pink1 & GGCAATGAAGATGATGTGGAAC & GGTCGGCAGGACATCAGGA & \\
\hline mao-b & CGTACATTGGACCAACTCAAAA & CCTCCAGAGGTTGTTGTAGTCC & Sarath Babu et al., 2016 \\
\hline
\end{tabular}


eGFP + and mCherry+ cells, with $n=6$ and 4 , respectively, were analyzed using a two-way ANOVA with multiple comparisons. eGFP+ multiple comparisons were performed using the Sidak method, while mCherry + multiple comparisons used the HolmSidak method. Statistical significance was determined using a 95\% confidence interval where $p<0.05$. ${ }^{*} p<0.05,{ }^{* *} p<0.01$, *** $p<0.001$, and ${ }^{* * * *} p<0.0001$.

\section{RESULTS}

\section{Cerebroventricular Microinjection Optimization and the LC50 Dose for MPTP}

Cerebroventricular microinjection, based on the protocol of Kizil and Brand (2011), was performed on 10-month old adult $\operatorname{Tg}($ dat:eGFP) and $\operatorname{Tg}$ (dat:tom20 MLS:mCherry) zebrafish. A range of MPTP concentrations was administered to 8 fish in each group to determine the sublethal dose. The range comprised of $10,25,35$, and $100 \mathrm{mM}$ concentrations of MPTP. Injections were performed daily for four consecutive days. Mortality rates were quantified following each injection point. A concentration of $100 \mathrm{mM}$ was shown to be completely lethal following the last injection, whereas $35 \mathrm{mM}$ resulted in 50\% survival. Both 10 and $25 \mathrm{mM}$ did not result in any observed death, thus $25 \mathrm{mM}$ was determined to be the working dose for the experiments performed in this study (Figure 1A).

\section{Effect of MPTP on Dopaminergic Neurons}

One common discrepancy in PD literature is the correlation between tyrosine hydroxylase (TH) and dopaminergic neurons. Although a widely accepted proxy, $\mathrm{TH}$ is involved in the biosynthesis of epinephrine in addition to dopamine. Here we performed injections on $\operatorname{Tg}($ dat:eGFP) zebrafish to analyze eGFP signal, providing a more direct assessment of dopaminergic degeneration as dat expression is localized specifically to dopaminergic neurons.

1-Methyl-4-phenyl-1,2,3,6-tetrahydropyridine was shown to impact dopaminergic neurons in three prominent regions within the zebrafish brain (Figure 1B); the olfactory bulbs (OBs; Figures 1C,D), telencephalon (Figures 1E,F) and periventricular pretectal nucleus (PPv; Figures 1G,H). The more immediate effects of MPTP were observed in the OBs with 2 and $38 \%$ reductions in eGFP+ cells following the first and second injections, respectively. In the OBs, this loss continued with each injection as MPTP-injected zebrafish resulted in losses of 32 and $47 \%$ following the third and fourth injections (Figure 1I). Likewise, MPTP-injected fish displayed reductions up to $41 \%$ in the telencephalon following the same injection regimen (Figure 1J). These effects were less severe within the PPv where a $25 \%$ loss of eGFP+ cells was observed following the final injection (data not shown).

To consolidate these findings at the genetic level, global brain transcripts for dat and th1 were quantified using qRTPCR. We found that both dat and th1 expression levels decreased by 40 and 20\%, respectively in MPTP-injected zebrafish (Figure 1K). Interestingly, we found 71 and 35\% increases in sox 2 and nestin gene expression following the fourth injection point suggesting immediate regenerative activation following dopaminergic chemo-ablation (Figure $\mathbf{1 K}$ ). To investigate potential pathways involved, we analyzed shha (sonic hedgehog a) expression, as shha has been identified as a key regulator of dopaminergic embryonic neurogenesis in zebrafish and other vertebrates (Hynes et al., 1995; Wullimann and Umeasalugo, 2020). We found a $29 \%$ increase in sha expression in MPTPinjected zebrafish; however, this increase did not reach statistical significance (Figure 1K). Our findings demonstrate the CVMI delivery of MPTP to induce considerable dopaminergic cell death and that there is a rapid neurogenic signaling response following the last injection.

\section{Effect of MPTP on Dopaminergic Mitochondria}

To assess the effects of MPTP on dopaminergic mitochondrial morphology, we performed injections in $\operatorname{Tg}$ (dat:tom 20 MLS:mCherry) zebrafish that label the mitochondria of dopaminergic neurons with mCherry (Noble et al., 2015). Following the last injection, cryosections were stained with dsRed, an antibody that recognizes mCherry, to visualize any potential disequilibrium between fission and fusion (Figure 2A). The OBs were selected for assessment due to their involvement in both sensory and motor function. We observed a significant increase in fragmented mitochondria of the MPTP-injected zebrafish relative to the PBS control (Figures 2B,C). Thus, 65\% of all mitochondria analyzed in the MPTP-injected fish were fragmented, compared to only $8 \%$ in controls. Inversely, $4 \%$ of mitochondrial displayed a fused morphology in the MPTP-treated fish, compared to $39 \%$ in controls (Figure 2D).

To further support these findings, we analyzed genes associated with mitochondrial maintenance, structure and function. Most notable are increases observed in genes involved in mitophagy, where pink1 is increased by $63 \%$ and parkin mRNA levels reach approximately four-times of those levels seen in controls. Consistent with the fragmented phenotype observed through IHC, fis1 (mitochondrial fission 1) transcript levels were significantly increased by $53 \%$, whereas $m f n 1$ (mitofusion 1) expression remained unaffected (Figure 2E). Moreover, we analyzed opa1 (optic dominant atrophy 1) as it is involved in regulating mitochondrial cytochrome$c$ release and found expression to remain unchanged. The apoptotic marker p53 was also analyzed and revealed that there was no effect of MPTP on this mechanism of cell death. Together these data suggest MPTP induces cell death in dopaminergic neurons via a mitophagy directed mechanism with mitochondrial fragmentation.

\section{Effect of MPTP on Zebrafish Behavior}

To determine if the observed neurotoxicity translates into a locomotor or sensory phenotypes, zebrafish activities were monitored following every injection point and after a 2-week 

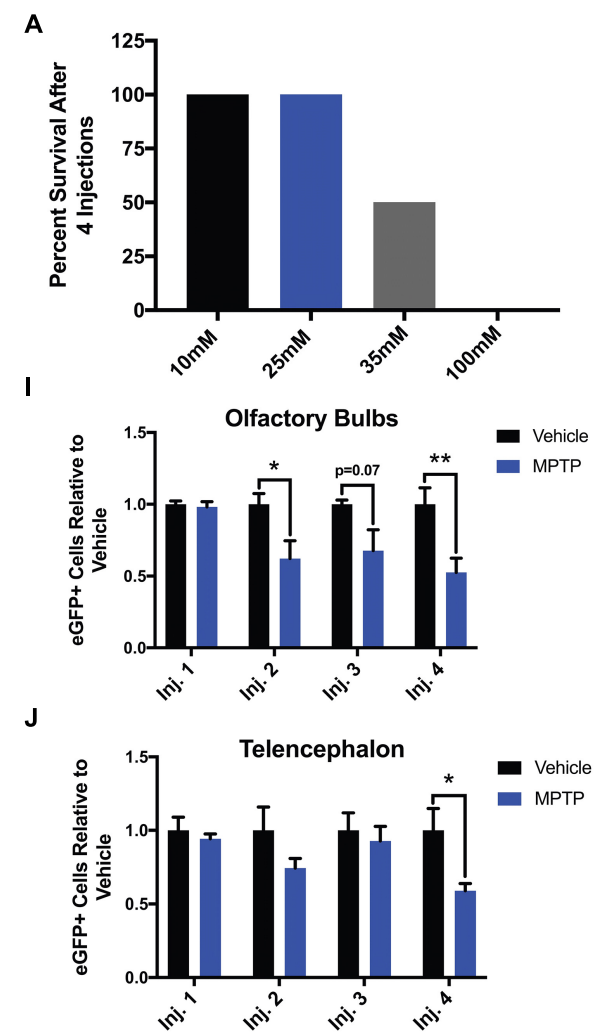

K

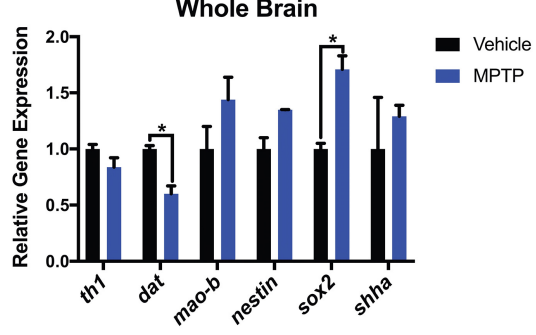

B
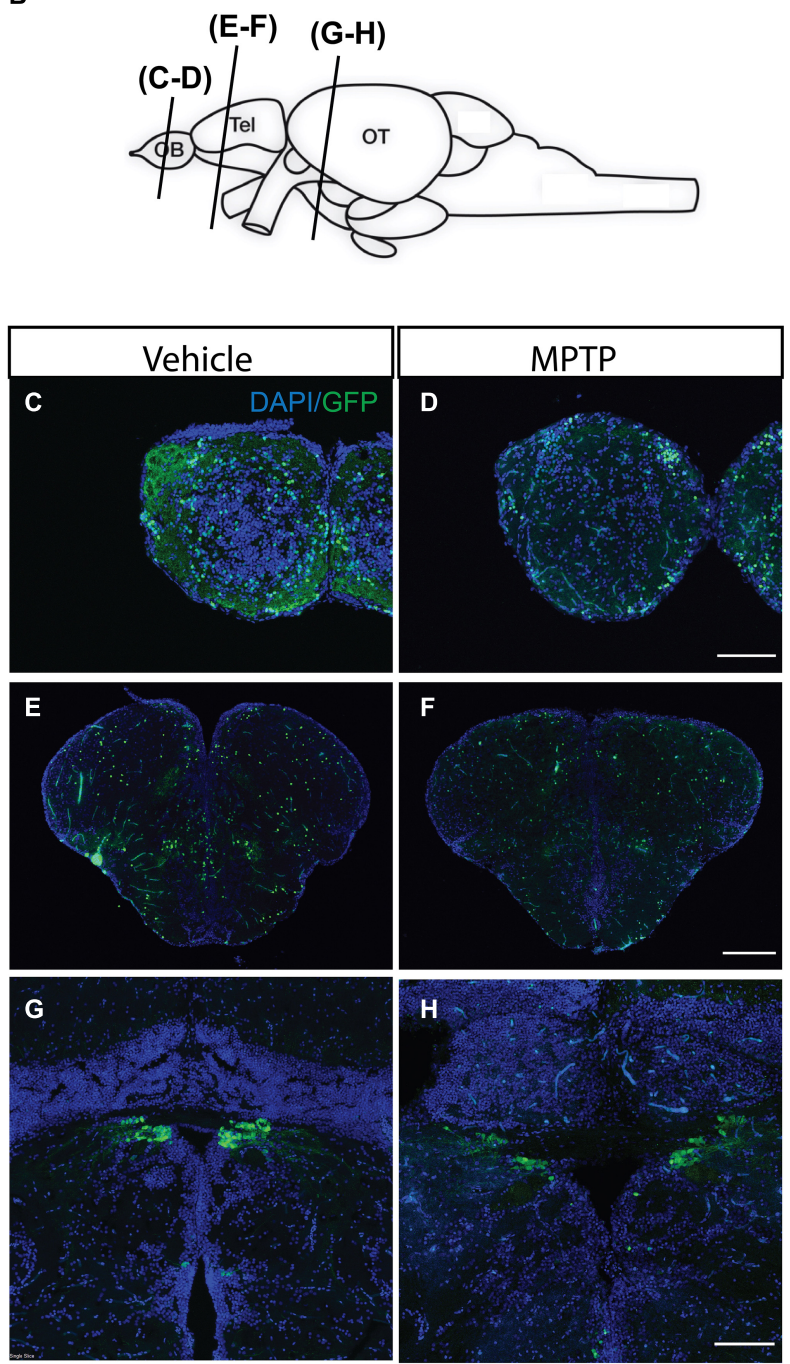

$\mathrm{H}$

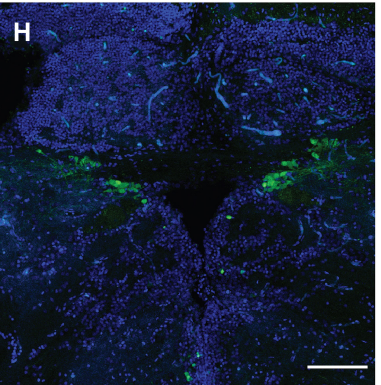

FIGURE 1 | Impact of MPTP on various brain regions of adult Tg(dat:eGFP) zebrafish. (A) MPTP dose-response. (B) Schematic representation of the zebrafish brain with the planes of section indicated. Immunohistochemical labeling of eGFP (green) and DAPI (blue) to observe dopaminergic neurons in the olfactory bulbs (OBs) $\mathbf{( C , D )}$, telencephalon (E,F), and periventricular pretectal nucleus (PPV) $\mathbf{( G , H ) . ~ ( I , J ) ~ Q u a n t i f i c a t i o n ~ o f ~ e G F P + ~ c e l l s ~ w i t h i n ~ t h e ~ O B s ~ a n d ~ t e l e n c e p h a l o n ~ r e s p e c t i v e l y . ~}$ $(n=6)$. (K) Whole brain qRT-PCR analysis for dopaminergic, and regeneration-related genes; th1, dat, sox2, nestin, and shha. ( $n=3$ whole brains from each respective group). Bars represent the mean \pm the SEM. ${ }^{\star} p<0.05,{ }^{\star \star} p<0.01$. Scale bars $=100$ and $200 \mu \mathrm{m}$ for the OB/PPV and telencephalon respectively. OB, olfactory bulbs; Tel, telencephalon; OT, optic tectum.

recovery period. The same individuals were analyzed throughout the experiment to delineate the effects of MPTP from individual variability within the population.

The locomotion parameters that were examined included the total distance traveled, average velocity and freezing bout (inactivity) duration. MPTP-injected zebrafish elicited 46.7 and $47 \%$ reductions in total distance and average velocity relative to the controls following the fourth injection (Figures 3B,C). Inversely, freezing activity increased gradually with each injection and a $51 \%$ increase was observed following the fourth injection (Figure 3A). Notably, all swimming patterns returned to normal following a 2-week recovery representative path images shown in Figures 3D-F.
Due to the substantial loss of dopaminergic neurons within the olfactory region, we sought to determine if this translates into any functional impairment of the olfactory response. To address this, we performed a repulsive stimulus test where cadaverine is administered to the arm of the apparatus the zebrafish is residing in. From quantifying the ratio of time spent in the stimulus arm, we found that cadaverine did not provoke a rapid repulsion response in MPTPinjected fish as observed in the controls. Control zebrafish spent $8 \%$ of the total time in the stimulus arm, whereas MPTP-injected zebrafish spent 19\%, more than double the amount of the time in that arm (Figure 4A). Similarly to swimming activities, these effects were shown to return 

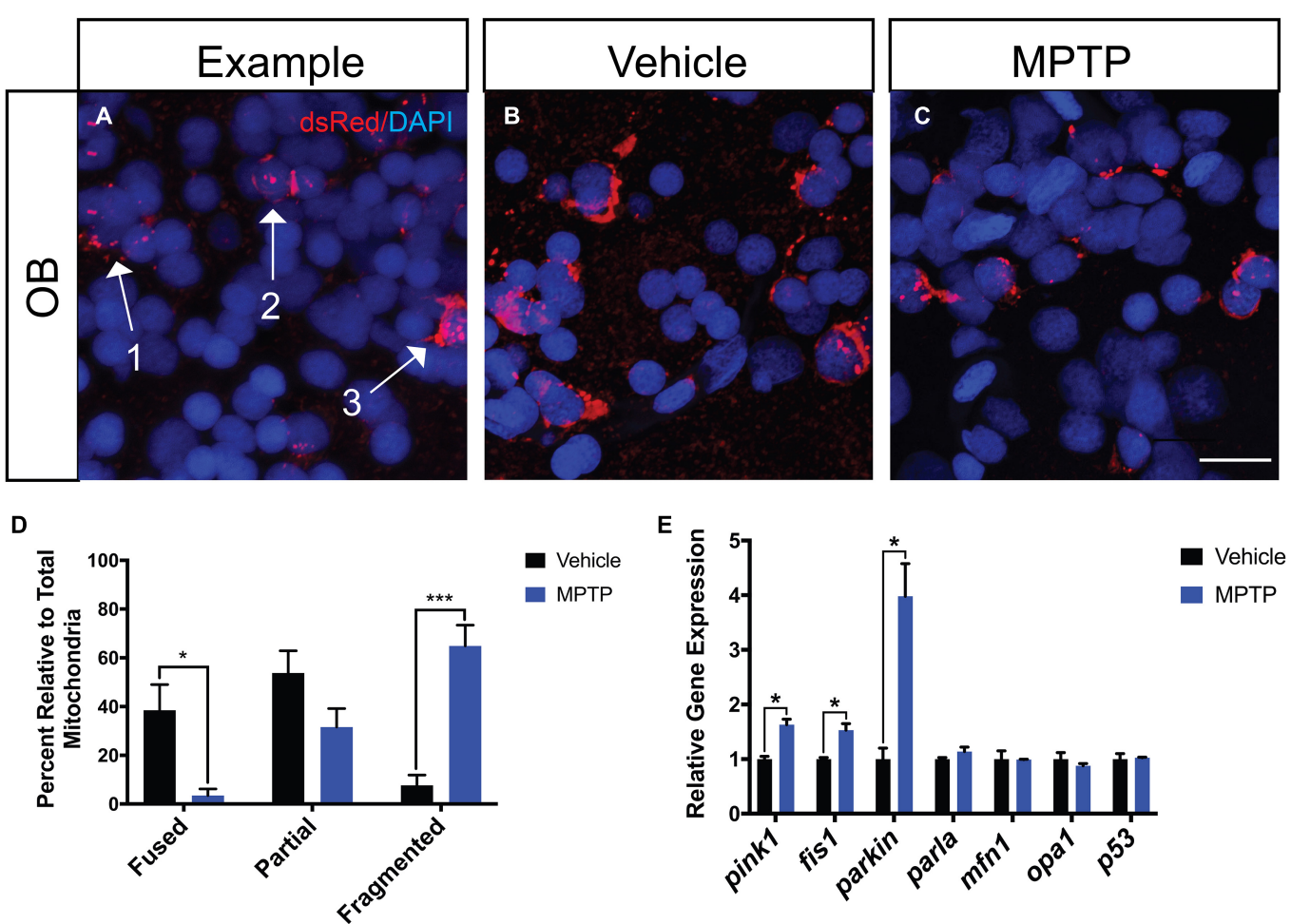

FIGURE 2 | Effect of MPTP on mitochondrial fission-fusion dynamics in Tg(dat:tom20 MLS:mCherry) adult zebrafish. (A) Representative image depicting fragmented mitochondria (Arrow 1), partially fragmented mitochondria (Arrow 2), and fused mitochondria (Arrow 3). (B,C) Immunohistochemical labeling marked mCherry+ for mitochondrial visualization and nuclei with DAPI+ on coronal OB cryosections from vehicle and MPTP injected zebrafish. (D) Proportion of fused, partially fragmented and fragmented mitochondria $(n=4)$. (E) Whole brain qRT-PCR analysis for mitochondrial and apoptotic genes; mao- $b$, pink1, fis 1, parkin, parla, mfn 1, opa1, and $p 53$ ( $n=3$ whole brains from each respective group). Scale bar $=10 \mu \mathrm{m}$. Bars represent the mean \pm the SEM. ${ }^{\star} p<0.05,{ }^{\star \star \star} p<0.001$.

to normal 2-weeks post injection representative path images shown in Figure 4B. Together these data suggest that dopaminergic ablation via CVMI impairs the olfactory response and perturbs motor function.

\section{DISCUSSION}

\section{The Efficiency of CVMI for Dopaminergic Cell Death}

Techniques to administer drugs will vary depending on the nature of the drug, the site of action and properties of the experimental animal. Popular administration methods include direct immersion, intraabdominal/IP and IM injections. However, each method comes with evident pitfalls. The largest downfall to IP injections is the primary route of absorption being through mesenteric vessels. Dosages are generally increased in this case to overcome the potential of the drug undergoing hepatic metabolism prior to systemic circulation (Abu-Hijleh et al., 1995; Turner et al., 2011). IM injections are commonly performed in mammalian models and are more difficult on zebrafish due to the reduced muscle mass and individual variability that poses the risk of differing drug absorption rates based on blood flow velocity (Manor and Sadeh, 1989; Dowd, 2017; Abbate et al., 2018). One must also be selective with the compound to be administered due to the potential for irritation and/or damage to the periphery nervous system that may result in paresis, paralysis or muscle necrosis (Rasmussen, 1978). Similarly, the direct immersion method of drug delivery makes it difficult to assess the exact quantity of drug being absorbed and can cause variability between treatments groups due to differing sizes, swimming patterns, and opercular movement speed (Cassar et al., 2020). The implementation of CVMI used in this study to administer MPTP did not result in any observable morphological abnormality nor systemic toxicity as observed using alternative methods (Bretaud et al., 2004; Kalyn et al., 2019).

1-Methyl-4-phenyl-1,2,3,6-tetrahydropyridine is commonly known as the "gold standard" in Parkinsonism studies due to the selective targeting of dopaminergic neurons. Alternative compounds may also act on other essential neuronal subtypes when injected in the absence of uptake inhibitors, such as 6-OHDA acting on both dopaminergic and epinephrinergic neurons (Fulceri et al., 2006; Bové and Perier, 2012). In zebrafish, the vast majority of MPTP studies were performed on embryos and/or larvae. However, these studies have laid the groundwork and outlined that MPTP affects ascending TH + diencephalic (5, 6, and 11) and pretectal clusters (7) more severely than others, while also decreasing dat and th1 transcript and neurotransmitter levels (Sallinen et al., 2009; Kalyn et al., 2019). Moreover, 
A
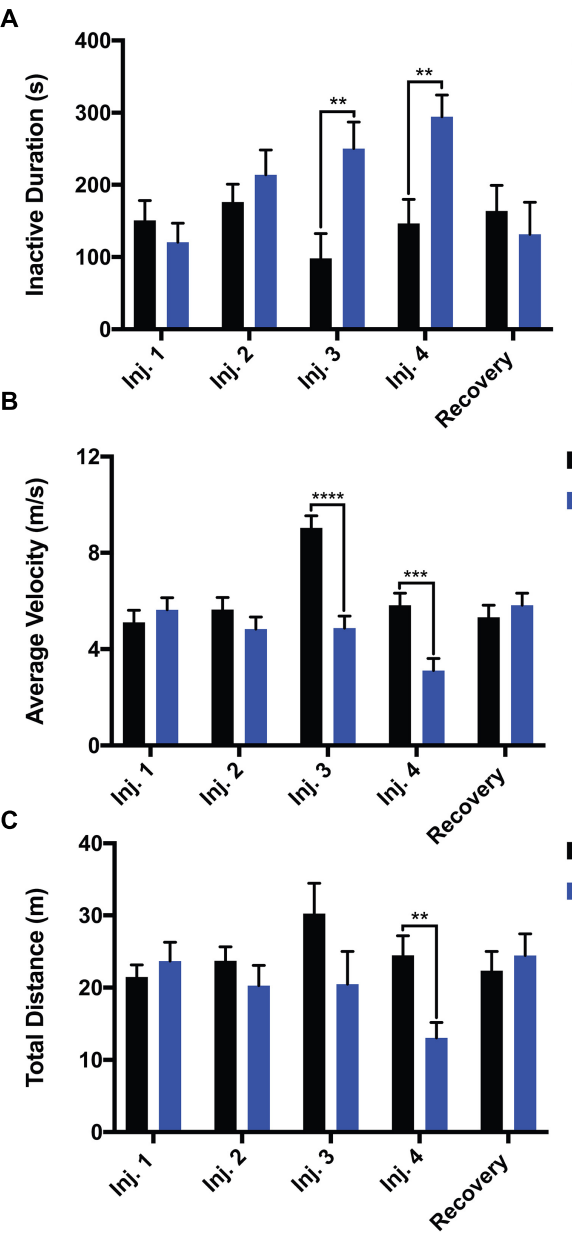

D

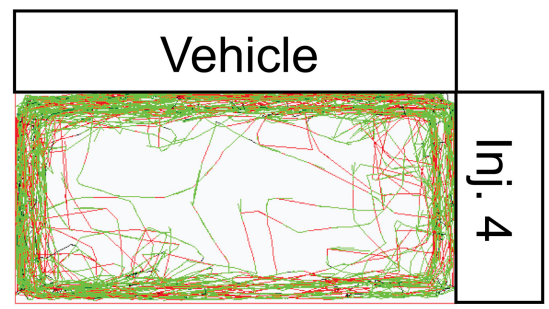

E

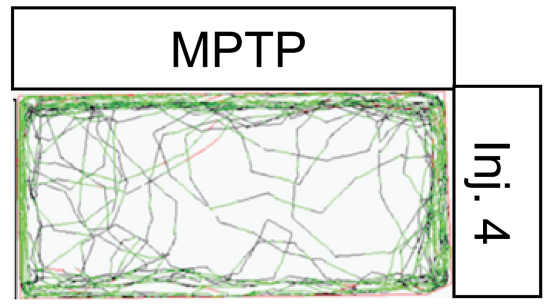

$\mathbf{F}$

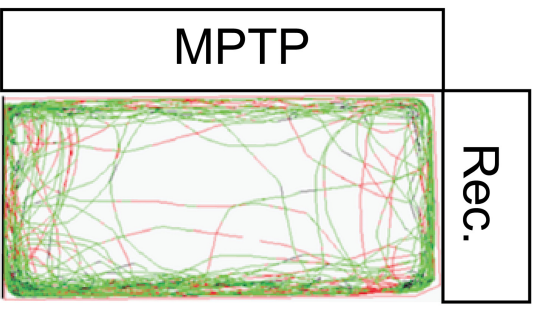

FIGURE 3 | Impact of MPTP on adult zebrafish swimming behavior. Fish were acclimated to the apparatus for 15 min prior to behavioral analyses. Seven-minutes trials were performed. (A-C) Analysis of inactive durations (proxy for freezing bouts), average velocity and total distance for MPTP injected zebrafish and vehicle controls. Respective path images are as follows; injection 4 vehicle control (D), injection 4 MPTP (E), and 2-week recovered MPTP treated zebrafish (F). Red lines represent fast movement, green lines represent slow movement and black lines represent inactive movement below the slow threshold $(n=10$ for all groups). Bars represent the mean \pm the SEM. ${ }^{\star \star} p<0.01,{ }^{\star \star \star} p<0.001$, and ${ }^{\star \star \star \star} p<0.0001$.
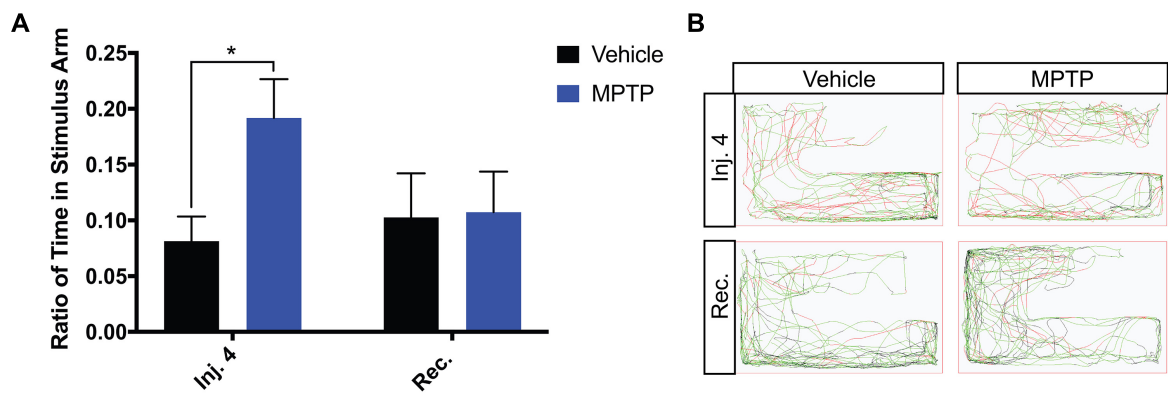

FIGURE 4 | Impact of MPTP on olfactory response in adult zebrafish. (A) Ratio of time spent in the stimulus arm following the fourth injection and 2-week recovery. (B) Respective path images for MPTP injected zebrafish and vehicle controls after the fourth injection and a 2-week recovery. Red lines represent fast movement, green lines represent slow movement and black lines represent inactive movement below the slow threshold. Bars represent the mean \pm the SEM. ${ }^{*} p<0.05$.

MPTP and MPP + have been shown to translate into motor perturbing phenotypes in $3,5,6$, and $7 \mathrm{dpf}$ zebrafish (Lam et al., 2005; Sallinen et al., 2009; Díaz-Casado et al., 2016;
Kalyn et al., 2019; Zhao et al., 2020). Larval studies have also identified a variety of compounds that can be used in a pretreatment exposure to mitigate some degree of MPTP induced 
dopaminergic neurodegeneration. A few of these compounds include deprenyl, melatonin and rosmarinic acid which act to alleviate stress through MAO-B inhibition, anti-oxidation and/or anti-inflammation (Parng et al., 2006; Sallinen et al., 2009; DíazCasado et al., 2016; Zhao et al., 2020). In adults, reports primarily consist of behavioral assessment without much examination of dopaminergic neurons and their mitochondria (Flinn et al., 2008; Selvaraj et al., 2019). However of the few that have investigated dopaminergic health, Anichtchik et al. (2004) have shown that a systemic injection of MPTP resulted in decreases in dopamine and noradenaline concentrations in the brain without any effect on TH immunoreactivity, TH co-localization with TUNEL or any increase in caspase-3 activity. Moreover, Bretaud et al. (2004) did not observe any dopaminergic neuron ablation following IP injections of MPTP, rotenone, or paraquat (other toxins implicated in PD studies). Here we report that CVMI was a successful method to deliver MPTP into the adult zebrafish brain through the observed increases in dopaminergic cell death within the telencephalic and olfactory regions of the adult brain, as well as, the spatial disorganization and slight reduction of dopaminergic neurons in the $\mathrm{PPv}$.

To assess the impact on dopaminergic neurons and further support our findings, th 1 , mao-b, and dat gene expression profiles were evaluated. Expression of th1 was analyzed in contrast to th 2 as th 2 is exclusively expressed in non-mammalian vertebrates and present in only four hypothalamic neuronal clusters within the hypothalamic and posterior tubercular regions of the brain (Barrios et al., 2020). The increased MAO-B mRNA expression observed suggests the continuous conversion of MPTP into its active toxic form MPP+ even following the fourth injection point. MPP+ then exerts its effects on cells expressing th1 and dat, as shown through decreases in respective gene mRNA levels. The $>50 \%$ dat expression remaining can be explained by the remaining dopaminergic neurons expressing dat as a means to recycle synaptic dopamine into the cytosol of dopaminergic neurons (Shimizu and Prasad, 1991; Giros et al., 1992; Sulzer et al., 2016; Merhi et al., 2021). Although we cannot rule out the possibility of a transient downregulation in dat/th1 expression that may contribute to the functional impairment and recovery of zebrafish behavior, the effect of MPTP on tom 20 expressing dopaminergic neurons combined with the eGFP data are suggestive of neurodegeneration occurring.

Interestingly, increases in expression for neural proliferative genes and shha were observed immediately following the last injection of MPTP. Shh is a main signaling morphogen in the ventralization of vertebrate neural tubes (Hynes et al., 1995; Wang et al., 2009; Carballo et al., 2018). Particularly in zebrafish, shha expressing cells have been shown to give rise to preglomerular complex and posterior tubercular dopaminergic neurons (Wullimann and Umeasalugo, 2020). Given the activity of Shh in embryonic dopaminergic neurogenesis and the increase in mRNA transcript levels observed in the present study, further analysis of Shh is warranted to determine its precise role in adult dopaminergic neurogenesis following ablation.

\section{Mitochondrial Response to MPTP in Dopaminergic Neurons}

Mitochondrial dysfunction is a hallmark of neurodegeneration in PD patients. There are various processes (fission, biogenesis, and mitophagy) that regulate mitochondrial quality within all cell types (Kobayashi et al., 2020). Fission is a mechanism that activates mitophagy and plays an adaptive role in the clearance of damaged mitochondria in response to oxidative stress or injury. Mitophagy is the process by which fragmented mitochondria are sequestered and degraded by lysosomes (Ding and Yin, 2012). Regarding biogenesis, it is well characterized that mitochondria provide energy to the cell through two dominant pathways: glycolysis and oxidative phosphorylation (Westermann, 2012). The variations in energetic supply or metabolic cues can also influence mitochondrial morphology (Yu and Pekkurnaz, 2018). Glycolytic mitochondria generally display a fragmented appearance with fission contributing to mitochondrial proliferation, while mitochondria undergoing oxidative phosphorylation display a more fused conformation (Rossignol et al., 2004; Westermann, 2012; Youle and van der Bliek, 2012; Seo et al., 2018). Dependent on the energetic requirements, highly active cells commonly rely on oxidative phosphorylation (Westermann, 2012). Dopaminergic neurons fall into this category.

Many studies have outlined the mechanism of MPTP mediated cell death via the inhibition of the ETC (Przedborski et al., 2000, 2004; Keane et al., 2011). However, a knowledge gap remains in visualizing these effects on mitochondrial conformational changes in the dopaminergic neurons of an adult zebrafish brain. Through performing injections on $\operatorname{Tg}$ (dat:tom 20 MLS:mCherry) zebrafish, we were able to observe the direct consequences of MPTP on mitochondrial morphology. Twentyfour hours following the fourth injection, MPTP-injected zebrafish exhibited significant fragmentation in dopaminergic neurons. Consistent with the results indicative of mitochondrial fission, there are significant increases in genes associated with this phenomenon: fis1 (mitochondrial fission protein 1), parkin and pink1 PTEN-induced kinase 1). The Parkin/Pink1 pathway is active in all mitochondria of healthy cells to expel dysfunctional or damaged mitochondria through mitophagy. Thus, damaged mitochondria in highly energetic cells will display a fragmented morphology. Addressing this phenomenon, Park et al. (2019) found increased numbers of abnormal mitochondria within dopaminergic neurons substantia nigra in macaques following MPTP exposure. However, this study outlined the mechanism of fission through Drp1 and Fis1 activation and not necessarily the subsequent cell death. Taken together with the decrease in eGFP+ expressing cells in our results, it is possible that the remaining dopaminergic neurons are degenerating via a mitophagy dominant mechanism inducing mitochondrial fission and neuronal death.

In addition to fragmentation as a response of oxidative stress and given that cellular bioenergetic demand can influence mitochondrial morphology, we cannot disregard that some dopaminergic neurons may be undergoing metabolic reprogramming from oxidative phosphorylation to aerobic 
glycolysis following MPTP injections. Emerging studies have shown that similar modifications in mitochondrial networking and structure occur as a mechanism to bioenergetically adapt to stress and/or inflammation (Baker et al., 2014). Nair et al. tested this hypothesis by inducing fission and energetic reversion to glycolysis through lipopolysaccharide (LPS) exposure in microglia. They subsequently found that a Drp1 protein inhibitor, Mdivi-1, attenuates reactive oxygen species (ROS) production, fragmentation and proinflammatory responses following LPS treatment in vivo and in vitro (Nair et al., 2019). Further analysis of genes and/or proteins involved in the present study must be done to support this possibility of dopaminergic mitochondrial metabolic reprogramming. One mechanism of interest was suggested by Zheng et al. (2016) who observed a loss of expression from lactate dehydrogenase and hexokinase, in parallel to the conversion in pyruvate kinase splicing from PKM2 to PKM1, that efficiently labels the transition from aerobic glycolysis in progenitor cells to oxidative phosphorylation in maturing neurons.

\section{Behavioral Consequence Following MPTP Injections}

The dopaminergic system is critical to several biological functions that include reward, pleasure, movement, and learning. Incidentally, the consequence of ablating dopamine producing cells can result in severe behavioral phenotypes. Microinjections of MPTP are shown to affect the dopaminergic projectome that modulates movement through observed impaired swimming parameters. MPTP-injected fish exhibited increased freezing bout duration, concurrent with decreases in both total distance and average velocity relative to the respective controls, resembling bradykinetic and dyskinetic symptoms (Vaz et al., 2018). MPTPinjected zebrafish displayed impaired olfactory response to the repulsive stimulus cadaverine. With the telencephalic and olfactory regions being two epicenters, in the zebrafish brain, that modulate behavior, this impact can be explained by the drastic decreases in eGFP+ cells residing within the OBs and telencephalon in MPTP-injected zebrafish. These effects are greater than those observed in our group's previous work from Godoy et al. (2020), that used a chemogenetic approach to ablate dopaminergic neurons. The phenotypes observed in this study were transient as all motor and sensory functions were restored following a 2 -week recovery period. This capacity for regeneration alone makes the zebrafish advantageous in neurodegenerative studies, however, this also poses a hindrance toward long-term therapeutic studies as it may be difficult to delineate any restorative effects between the therapeutic compound administered and the natural regeneration that occurs. Therapeutic studies using this model commonly treat the zebrafish prior to neuronal insult to evaluate the efficiency of varying neuroprotective compounds. Given the 2-week transiency of sensorimotor phenotypes, it would be beneficial to implement a short-term regenerative study following ablation to identify possible neurogenic drivers of dopaminergic neurons in adult zebrafish to gain a better temporal understanding of behavioral restoration. As previously mentioned, identifying drivers, such as Shh, may serve as a foundation for gene-therapy application in higher vertebrate models.

\section{CONCLUSION}

Literature surrounding PD suffers from discrepancies among the degree of dopaminergic ablation and behavioral phenotypes resulting from neurotoxin treatments. Here, we demonstrated that CVMI delivery of MPTP induces substantial dopaminergic neuron death within the telencephalon and olfactory regions of the adult zebrafish brain. This reduction in dopaminergic neurons resulted in transient sensory and motor phenotypes. Moreover, using the $\operatorname{Tg}$ (dat:tom 20 MLS:mCherry) zebrafish, we were able to observe a significant increase in the proportion of fragmented mitochondria in MPTP-injected zebrafish. Supporting gene expression evidence suggests that the dopaminergic cell death is activated via a mitophagy mechanism that results in increased fission and a more fragmented mitochondrial morphology. Taken together this study will ameliorate our understanding of chemically induced mitochondrial dysfunction in the neurodegeneration of dopaminergic neurons and other PD pathologies.

\section{DATA AVAILABILITY STATEMENT}

The original contributions presented in the study are included in the article/supplementary material, further inquiries can be directed to the corresponding author.

\section{ETHICS STATEMENT}

The animal study was reviewed and approved by the University of Ottawa Animal Care Committee.

\section{AUTHOR CONTRIBUTIONS}

MK and ME: conceptualization, methodology, validation, and writing - review and editing. MK: data curation, formal analysis, supervision, visualization, and writing - original draft. Both authors have read and agreed to the published version of the manuscript.

\section{FUNDING}

This research was supported by the Natural Sciences and Engineering Research Council of Canada, grant number 121795.

\section{ACKNOWLEDGMENTS}

We would like to thank Dung Ngo for his help in the conceptualization of this study and Gary Hatch for critical reading of the manuscript. We would also like to thank Dana Elsaid and Erin Fletcher for their help in cell counting. 


\section{REFERENCES}

Abbate, A., Almasio, P. L., Mongitore, M., Di Vita, G., and Patti, R. (2018). Necrotizing soft tissue fasciitis after intramuscular injection. Case Rep. Surg. 2018:3945497. doi: 10.1155/2018/3945497

Abu-Hijleh, M. F., Habbal, O. A., and Moqattash, S. T. (1995). The role of the diaphragm in lymphatic absorption from the peritoneal cavity. J. Anat. $186(\mathrm{Pt}$ 3), 453-467.

Anichtchik, O. V., Kaslin, J., Peitsaro, N., Scheinin, M., and Panula, P. (2004). Neurochemical and behavioural changes in zebrafish Danio rerio after systemic administration of 6-hydroxydopamine and 1-methyl-4-phenyl1,2,3,6-tetrahydropyridine. J. Neurochem. 88, 443-453. doi: 10.1111/j.14714159.2004.02190.x

Baker, B., Maitra, U., Geng, S., and Li, L. (2014). Molecular and cellular mechanisms responsible for cellular stress and low-grade inflammation induced by a super-low dose of endotoxin. J. Biol. Chem. 289, 16262-16269. doi: 10.1074/ jbc.M114.569210

Barreto-Valer, K., López-Bellido, R., Macho Sánchez-Simón, F., and Rodríguez, R. E. (2012). Modulation by cocaine of dopamine receptors through miRNA133b in Zebrafish embryos. PLoS One 7:e52701. doi: 10.1371/journal.pone. 0052701

Barrios, J. P., Wang, W. C., England, R., Reifenberg, E., and Douglass, A. D. (2020). Hypothalamic dopamine neurons control sensorimotor behavior by modulating brainstem premotor nuclei in Zebrafish. Curr. Biol. 30, 4606.e44618.e4. doi: 10.1016/j.cub.2020.09.002

Bonifati, V., Rizzu, P., van Baren, M. J., Schaap, O., Breedveld, G. J., Krieger, E., et al. (2003). Mutations in the DJ-1 gene associated with autosomal recessive early-onset parkinsonism. Science 299, 256-259. doi: 10.1126/science.107 7209

Bové, J., and Perier, C. (2012). Neurotoxin-based models of Parkinson's disease. Neuroscience 211, 51-76. doi: 10.1016/j.neuroscience.2011.10.057

Bretaud, S., Lee, S., and Guo, S. (2004). Sensitivity of zebrafish to environmental toxins implicated in Parkinson's disease. Neurotoxicol. Teratol. 26, 857-864. doi: 10.1016/j.ntt.2004.06.014

Carballo, G. B., Honorato, J. R., de Lopes, G. P. F., and Spohr, T. C. L. (2018). A highlight on Sonic hedgehog pathway. Cell Commun. Signal. 16:1.

Cassar, S., Adatto, I., Freeman, J. L., Gamse, J. T., Iturria, I., Lawrence, C., et al. (2020). Use of zebrafish in drug discovery toxicology. Chem. Res. Toxicol. 33, 95-118. doi: 10.1021/acs.chemrestox.9b00335

Chen, Y.-C., Semenova, S., Rozov, S., Sundvik, M., Bonkowsky, J. L., and Panula, P. (2016). A novel developmental role for dopaminergic signaling to specify hypothalamic neurotransmitter identity. J. Biol. Chem. 291, 21880-21892. doi: 10.1074/jbc.M115.697466

Cheng, H.-C., Ulane, C. M., and Burke, R. E. (2010). Clinical progression in Parkinson disease and the neurobiology of axons. Ann. Neurol. 67, 715-725. doi: 10.1002/ana.21995

Dauer, W., and Przedborski, S. (2003). Parkinson's disease: mechanisms and models. Neuron 39, 889-909. doi: 10.1016/S0896-6273(03)00568-3

Dawson, T. M., and Dawson, V. L. (2010). The role of parkin in familial and sporadic Parkinson's disease. Mov. Disord. 25(Suppl. 1), S32-S39. doi: 10.1002/ mds. 22798

de Lau, L. M. L., and Breteler, M. M. B. (2006). Epidemiology of Parkinson's disease. Lancet Neurol. 5, 525-535. doi: 10.1016/S1474-4422(06)70471-9

Dexter, D. T., and Jenner, P. (2013). Parkinson disease: from pathology to molecular disease mechanisms. Free Radic. Biol. Med. 62, 132-144. doi: 10.1016/ j.freeradbiomed.2013.01.018

Díaz-Casado, M. E., Lima, E., García, J. A., Doerrier, C., Aranda, P., Sayed, R. K., et al. (2016). Melatonin rescues zebrafish embryos from the parkinsonian phenotype restoring the parkin/PINK1/DJ-1/MUL1 network. J. Pineal Res. 61, 96-107. doi: 10.1111/jpi.12332

Ding, W.-X., and Yin, X.-M. (2012). Mitophagy: mechanisms, pathophysiological roles, and analysis. Biol. Chem. 393, 547-564. doi: 10.1515/hsz-2012-0119

Dowd, F. J. (2017). Pharmacokinetics: The Absorption, Distribution, and Fate of Drugs, Seventh Edn. Amsterdam: Elsevier Inc.

Du, Y., Guo, Q., Shan, M., Wu, Y., Huang, S., Zhao, H., et al. (2016). Spatial and temporal distribution of dopaminergic neurons during development in Zebrafish. Front. Neuroanat. 10:115. doi: 10.3389/fnana.2016.0 0115
Flinn, L., Bretaud, S., Lo, C., Ingham, P. W., and Bandmann, O. (2008). Zebrafish as a new animal model for movement disorders. J. Neurochem. 106, 1991-1997. doi: 10.1111/j.1471-4159.2008.05463.x

Fulceri, F., Biagioni, F., Lenzi, P., Falleni, A., Gesi, M., Ruggieri, S., et al. (2006). Nigrostriatal damage with 6-OHDA. Ann. N. Y. Acad. Sci. 1074, 344-348. doi: 10.1196/annals.1369.032

Funayama, M., Hasegawa, K., Kowa, H., Saito, M., Tsuji, S., and Obata, F. (2002). A new locus for Parkinson's disease (PARK8) maps to chromosome 12p11.2q13.1. Ann. Neurol. 51, 296-301. doi: 10.1002/ana.10113

Giros, B., El Mestikawy, S., Godinot, N., Zheng, K., Han, H., Yang-Feng, T., et al. (1992). Cloning, pharmacological characterization, and chromosome assignment of the human dopamine transporter. Mol. Pharmacol. 42, 383-390.

Godoy, R., Hua, K., Kalyn, M., Cusson, V.-M., Anisman, H., and Ekker, M. (2020). Dopaminergic neurons regenerate following chemogenetic ablation in the olfactory bulb of adult Zebrafish (Danio rerio). Sci. Rep. 10:12825. doi: 10.1038/s41598-020-69734-0

Hare, D. J., Adlard, P. A., Doble, P. A., and Finkelstein, D. I. (2013). Metallobiology of 1-methyl-4-phenyl-1,2,3,6-tetrahydropyridine neurotoxicity. Metallomics 5, 91-109. doi: 10.1039/C2MT20164J

Hatano, Y., Li, Y., Sato, K., Asakawa, S., Yamamura, Y., Tomiyama, H., et al. (2004). Novel PINK1 mutations in early-onset parkinsonism. Ann. Neurol. 56, 424-427. doi: 10.1002/ana.20251

Holzschuh, J., Ryu, S., Aberger, F., and Driever, W. (2001). Dopamine transporter expression distinguishes dopaminergic neurons from other catecholaminergic neurons in the developing zebra ${ }^{\circledR}$ sh embryo. Mech. Dev. 101, 237-243.

Hussain, A., Saraiva, L. R., Ferrero, D. M., Ahuja, G., Krishna, V. S., Liberles, S. D., et al. (2013). High-affinity olfactory receptor for the death-associated odor cadaverine. Proc. Natl. Acad. Sci. U.S.A. 110, 19579-19584. doi: 10.1073/pnas. 1318596110

Hynes, M., Porter, J. A., Chiang, C., Chang, D., Tessier-Lavigne, M., Beachy, P. A., et al. (1995). Induction of midbrain dopaminergic neurons by Sonic hedgehog. Neuron 15, 35-44. doi: 10.1016/0896-6273(95)90062-4

Kalyn, M., Hua, K., Mohd Noor, S., Wong, C. E. D., and Ekker, M. (2019). Comprehensive analysis of neurotoxin-induced ablation of dopaminergic neurons in Zebrafish larvae. Biomedicines 8:1. doi: 10.3390/biomedicines8010001

Keane, P. C., Kurzawa, M., Blain, P. G., and Morris, C. M. (2011). Mitochondrial dysfunction in parkinson\&\#39;s disease. Park. Dis. 2011:716871. doi: 10.4061/ $2011 / 716871$

Kitada, T., Asakawa, S., Hattori, N., Matsumine, H., Yamamura, Y., Minoshima, S., et al. (1998). Mutations in the parkin gene cause autosomal recessive juvenile parkinsonism. Nature 392, 605-608. doi: 10.1038/33416

Kizil, C., and Brand, M. (2011). Cerebroventricular Microinjection (CVMI) into adult Zebrafish brain is an efficient misexpression method for forebrain ventricular cells. PLoS One 6:e27395. doi: 10.1371/journal.pone.0027395

Kobayashi, S., Zhao, F., Zhang, Z., Kobayashi, T., Huang, Y., Shi, B., et al. (2020). Mitochondrial fission and mitophagy coordinately restrict high glucose toxicity in cardiomyocytes. Front. Physiol. 11:1596. doi: 10.3389/fphys.2020.604069

Lam, C. S., Korzh, V., and Strahle, U. (2005). Short communication Zebrafish embryos are susceptible to the dopaminergic neurotoxin MPTP. Eur. J. Neurosci. 21, 1758-1762. doi: 10.1111/j.1460-9568.2005.03988.x

Manor, D., and Sadeh, M. (1989). Muscle fibre necrosis induced by intramuscular injection of drugs. Br. J. Exp. Pathol. 70, 457-462.

Meredith, G. E., and Rademacher, D. J. (2011). MPTP mouse models of Parkinson's disease: an update. J. Parkinsons. Dis. 1, 19-33. doi: 10.3233/JPD-2011-11023

Merhi, R., Kalyn, M., Zhu-Pawlowsky, A., and Ekker, M. (2021). Loss of parla function results in inactivity, olfactory impairment, and dopamine neuron loss in Zebrafish. Biomedicines 9:205. doi: 10.3390/biomedicines 9020205

Muangpaisan, W., Mathews Mbbs, A., Hori, H., and Seidel Mphil, D. (2011). A systematic review of the worldwide prevalence and incidence of parkinson's disease. J. Med. Assoc. Thai. 94, 749-755.

Nair, S., Sobotka, K. S., Joshi, P., Gressens, P., Fleiss, B., Thornton, C., et al. (2019). Lipopolysaccharide-induced alteration of mitochondrial morphology induces a metabolic shift in microglia modulating the inflammatory response in vitro and in vivo. Glia 67, 1047-1061. doi: 10.1002/glia.23587

Noble, S., Godoy, R., Affaticati, P., and Ekker, M. (2015). Transgenic Zebrafish expressing mcherry in the mitochondria of dopaminergic neurons. Zebrafish 12, 349-356. doi: 10.1089/zeb.2015.1085 
Park, J., Seo, J., Won, J., Yeo, H.-G., Ahn, Y.-J., Kim, K., et al. (2019). Abnormal mitochondria in a non-human primate model of MPTP-induced Parkinson's disease: Drp1 and CDK5/p25 signaling. Exp. Neurobiol. 28, 414-424. doi: 10. 5607/en.2019.28.3.414

Parng, C., Ton, C., Lin, Y.-X., Roy, N. M., and McGrath, P. (2006). A zebrafish assay for identifying neuroprotectants in vivo. Neurotoxicol. Teratol. 28, 509-516. doi: 10.1016/j.ntt.2006.04.003

Przedborski, S., Jackson-Lewis Vernice, P., Djaldetti, R., Liberatore, G., Vila, M., Vukosavic, S., et al. (2000). The parkinsonian toxin MPTP: action and mechanism. Restor Neurol. Neurosci. 16, 135-142.

Przedborski, S., Tieu, K., Perier, C., and Vila, M. (2004). MPTP as a mitochondrial neurotoxic model of Parkinson's disease. J. Bioenerg. Biomembr. 36, 375-379. doi: 10.1023/B:JOBB.0000041771.66775.d5

Rasmussen, F. (1978). Tissue damage at the injection site after intramuscular injection of drugs. Vet. Sci. Commun. 2, 173-182. doi: 10.1007/BF02291447

Rossignol, R., Gilkerson, R., Aggeler, R., Yamagata, K., Remington, S. J., and Capaldi, R. A. (2004). Energy substrate modulates mitochondrial structure and oxidative capacity in cancer cells. Cancer Res. 64, 985-993. doi: 10.1158/00085472.can-03-1101

Sallinen, V., Torkko, V., Sundvik, M., Reenilä, I., Khrustalyov, D., Kaslin, J., et al. (2009). MPTP and MPP+ target specific aminergic cell populations in larval zebrafish. J. Neurochem. 108, 719-731. doi: 10.1111/j.1471-4159.2008.05793.x

Sarath Babu, N., Murthy, C. L. N., Kakara, S., Sharma, R., Brahmendra Swamy, C. V., and Idris, M. M. (2016). 1-Methyl-4-phenyl-1,2,3,6-tetrahydropyridine induced Parkinson's disease in zebrafish. Proteomics 16, 1407-1420. doi: 10. 1002/pmic.201500291

Selvaraj, V., Venkatasubramanian, H., Ilango, K., and Santhakumar, K. (2019). A simple method to study motor and non-motor behaviors in adult zebrafish. J. Neurosci. Methods 320, 16-25. doi: 10.1016/j.jneumeth.2019.03.008

Seo, B. J., Yoon, S. H., and Do, J. T. (2018). Mitochondrial dynamics in stem cells and differentiation. Int. J. Mol. Sci. 19:3893. doi: 10.3390/ijms19123893

Shimizu, I., and Prasad, C. (1991). Dopamine Transporter in Aging. Pergamon: Springer, 171-173.

Stewart, A. M., Braubach, O., Spitsbergen, J., Gerlai, R., and Kalueff, A. V. (2014). Zebrafish models for translational neuroscience research: from tank to bedside. Trends Neurosci. 37, 264-278. doi: 10.1016/j.tins.2014.02.011

Sulzer, D., Cragg, S. J., and Rice, M. E. (2016). Striatal dopamine neurotransmission: regulation of release and uptake. Basal Ganglia 6, 123-148.

Tang, R., Dodd, A., Lai, D., McNabb, W. C., and Love, D. R. (2007). Validation of Zebrafish (Danio rerio) reference genes for quantitative real-time RT-PCR normalization. Acta Biochim. Biophys. Sin. 39, 384-390. doi: 10.1111/j.17457270.2007.00283.x

Tieu, K. (2011). A guide to neurotoxic animal models of Parkinson's disease. Cold Spring Harb. Perspect. Med. 1:a009316. doi: 10.1101/cshperspect.a009316

Turner, P. V., Brabb, T., Pekow, C., and Vasbinder, M. A. (2011). Administration of substances to laboratory animals: routes of administration and factors to consider. J. Am. Assoc. Lab. Anim. Sci. 50, 600-613.

Van Kampen, J. M., McGeer, E. G., and Stoessl, A. J. (2000). Dopamine transporter function assessed by antisense knockdown in the rat: protection from dopamine neurotoxicity. Synapse 37, 171-178.
Vaz, R. L., Outeiro, T. F., and Ferreira, J. J. (2018). Zebrafish as an animal model for drug discovery in Parkinson's disease and other movement disorders: a systematic review. Front. Neurol. 9:347. doi: 10.3389/fneur.2018.00347

Wang, X., Mao, X., Xie, L., Greenberg, D. A., and Jin, K. (2009). Involvement of Notch1 signaling in neurogenesis in the subventricular zone of normal and ischemic rat brain in vivo. J. Cereb. Blood Flow Metab. 29, 1644-1654. doi: $10.1038 /$ jcbfm. 2009.83

Westermann, B. (2012). Bioenergetic role of mitochondrial fusion and fission. Biochim. Biophys. Acta Bioenerg. 1817, 1833-1838. doi: 10.1016/j.bbabio.2012. 02.033

Wullimann, M. F., and Umeasalugo, K. E. (2020). Sonic hedgehog expression in zebrafish forebrain identifies the teleostean pallidal signaling center and shows preglomerular complex and posterior tubercular dopamine cells to arise from shh cells. J. Comp. 528, 1321-1348. doi: 10.1002/cne.24825

Xi, Y., Yu, M., Godoy, R., Hatch, G., Poitras, L., and Ekker, M. (2011). Transgenic Zebrafish expressing green fluorescent protein in dopaminergic neurons of the ventral diencephalon. Dev. Dyn. 240, 2539-2547. doi: 10.1002/dvdy.22742

Youle, R. J., and van der Bliek, A. M. (2012). Mitochondrial fission, fusion, and stress. Science 337, 1062-1065. doi: 10.1126/science.1219855

Yu, S. B., and Pekkurnaz, G. (2018). Mechanisms orchestrating mitochondrial dynamics for energy homeostasis. J. Mol. Biol. 430, 3922-3941. doi: 10.1016/ j.jmb.2018.07.027

Zhao, Y., Han, Y., Wang, Z., Chen, T., Qian, H., He, J., et al. (2020). Rosmarinic acid protects against 1-methyl-4-phenyl-1,2,3,6-tetrahydropyridine-induced dopaminergic neurotoxicity in zebrafish embryos. Toxicol. Vitr. 65:104823. doi: 10.1016/j.tiv.2020.104823

Zheng, X., Boyer, L., Jin, M., Mertens, J., Kim, Y., Ma, L., et al. (2016). Metabolic reprogramming during neuronal differentiation from aerobic glycolysis to neuronal oxidative phosphorylation. eLife 5:e13374. doi: 10.7554/eLife.1 3374

Zimprich, A., Biskup, S., Leitner, P., Lichtner, P., Farrer, M., Lincoln, S., et al. (2004). Mutations in LRRK2 cause autosomal-dominant parkinsonism with pleomorphic pathology. Neuron 44, 601-607. doi: 10.1016/j.neuron.2004.11. 005

Conflict of Interest: The authors declare that the research was conducted in the absence of any commercial or financial relationships that could be construed as a potential conflict of interest.

Publisher's Note: All claims expressed in this article are solely those of the authors and do not necessarily represent those of their affiliated organizations, or those of the publisher, the editors and the reviewers. Any product that may be evaluated in this article, or claim that may be made by its manufacturer, is not guaranteed or endorsed by the publisher.

Copyright (c) 2021 Kalyn and Ekker. This is an open-access article distributed under the terms of the Creative Commons Attribution License (CC BY). The use, distribution or reproduction in other forums is permitted, provided the original author(s) and the copyright owner(s) are credited and that the original publication in this journal is cited, in accordance with accepted academic practice. No use, distribution or reproduction is permitted which does not comply with these terms. 